\title{
An Anomalous Story Of A Rare Percutaneous Intervention To Left Anterior Descending / Right Coronary Artery Bifurcation
}

\author{
Harinder Bali ${ }^{1}$, Amreen Dhindsa ${ }^{2}$, and Sankalp Bharti ${ }^{3}$ \\ ${ }^{1}$ Paras Hospital Panchkula \\ ${ }^{2}$ Paras Hospitals, Panchkula \\ ${ }^{3}$ Civil Hospital Panchkula
}

October 1,2020

\begin{abstract}
We report a rare percutaneous intervention to the Left Anterior Descending (LAD) and Right Coronary Artery (RCA) bifurcation in a 77 year old with anomalous origin of the RCA from mid LAD and Medina 1,1,1 bifurcation disease. The LAD/RCA bifurcation lesion was successfully treated using Mini crush technique.
\end{abstract}

\section{KEY WORDS:}

anomalous, bifurcation, percutaneous, intervention, guideliner

\section{KEY CLINICAL MESSAGE:}

Bifurcation lesions in anomalous coronary arteries are rare. Percutaneous intervention in such lesions is challenging and necessitates use of non standard hardware like GuideLiner catheter (Vascular Solutions,MN,USA) for adequate access and support.

\section{INTRODUCTION}

A single coronary artery arising from the Left Coronary sinus, with the Right Coronary Artery arising from the Left Anterior Descending Artery, is an extremely rare coronary artery anomaly. Usually benign, it may result in ischemia by various mechanisms including atherosclerotic involvement of the vessels, rendering a critical area of myocardium at risk. Percutaneous intervention in anomalous coronary arteries is particularly challenging. Use of non standard hardware may be required for adequate access and support. We describe a complex and rare percutaneous intervention to the Left Anterior Descending and Right Coronary Artery bifurcation in a 77 year old patient with anomalous origin of the Right Coronary Artery from mid Left Anterior Descending Artery and Medina 1,1,1 disease at the bifurcation. The LAD/RCA bifurcation lesion was successfully treated using the mini crush technique.

\section{CASE REPORT}

A 77 year-old male patient with coronary artery disease and a history of percutaneous intervention in LAD and LCx two years ago, was admitted with complaints of exertional angina. Co morbidities included hypertension and metastatic Hepatocellular Carcinoma.

ECG was normal. Echocardiography revealed hypokinesia of anterolateral and inferior wall. Trop I was 0.03 $\mathrm{pg} / \mathrm{ml}$. Coronary angiography was performed through right radial route using the $5 \mathrm{~F}$ Tiger catheter (Terumo Corporation, Tokyo, Japan). It revealed a normal left main coronary artery, a heavily calcific LAD with ostio-proximal patent LAD stent and diffuse 70-80\% stenosis in mid LAD, a dominant RCA with anomalous 
origin from mid LAD and ostial 90\% stenosis (Medina 1,1,1 disease at the mid LAD and anomalous RCA bifurcation). The LCx was diffusely diseased with patent mid LCx stent. Diffuse calcific 80-90\% stenosis in OM1 and and 50\% stenosis in OM2 was present. (Figure 1)

In view of symptomatic disease with a large area of myocardium at risk, a decision to revascularize was taken. Procedure was done through right femoral route using $7 \mathrm{~F}$ sheath. The left main artery was hooked with an XB3 7F (Cordis, USA) guiding catheter. A 0.14 inch BMW universal wire (Abbott Vascular, Santa Clara, USA) was used to cross the LAD lesion and was parked in distal LAD. The RCA lesion was then crossed with another 0.14 inch BMW universal wire (Abbott Vascular, Santa Clara, USA) and parked in the Posterior Descending Artery. Negotiating a balloon into the anomalous RCA was little challenging because of a sharp angle, necessitating the use of a $6 \mathrm{~F}$ GuideLiner catheter (Vascular Solutions, MN, USA). A 2.5x15mm Sprinter balloon (Medtronic Inc, MN, USA) was advanced into the anomalous RCA and predilatation of ostial anomalous RCA was done at 14 atmospheric pressure (Figure 2). The LAD lesion was predilated using a $2.5 \times 15 \mathrm{~mm}$ Sprinter balloon (Medtronic Inc, MN, USA). A Xience Xpedition 2.75x15mm stent (Abbott Vascular, Santa Clara, USA) was negotiated into the anomalous RCA using the 6F GuideLiner catheter. It was positioned in proximal RCA with slight protrusion into the LAD and deployed at 12 atmospheric pressure (Figure 3). This stent was then crushed with a $2.75 \times 18 \mathrm{~mm}$ Sprinter balloon (Medtronic Inc, MN, USA) (Figure 4). A $3 \times 23 \mathrm{~mm}$ Xience Xpedition stent (Abbott Vascular, Santa Clara, USA) was placed in mid LAD and deployed at 12 atmospheric pressure (Figure 5). Fielder XT wire (Asahi Intecc, Nagoya, Japan) was used to re cross RCA stent. Use of a $6 \mathrm{~F}$ GuideLiner catheter close to the origin of RCA ostium facilitated the negotiation of the balloon to the ostial RCA. Serial dilatation of ostium of RCA was done first with $1.5 \times 8 \mathrm{~mm}, 2.0 \times 10 \mathrm{~mm}$ and finally with $2.5 \times 10 \mathrm{~mm}$ Sprinter balloon (Medtronic Inc, MN, USA) (Figure 6). Final kissing balloon post dilatation of this bifurcation at mid LAD and RCA was done with two non compliant balloons, Voyager NC 3x15mm (Abbott Vascular, Santa Clara, USA) in mid LAD and Voyager NC 2.75x15mm (Abbott Vascular, Santa Clara, USA) in RCA (Figure 7). Proximal optimisation (POT) was done with a $3.5 \times 12 \mathrm{~mm}$ Voyager NC balloon (Abbott Vascular, Santa Clara, USA) at 20 atmospheric pressure. A satisfactory angiography result with TIMI 3 flow in both LAD and RCA was seen with no residual stenosis and no dissection (Figure 8). Patient made uneventful recovery and was discharged two days after procedure.

\section{DISCUSSION}

A single coronary artery arising from the Left Coronary sinus, with the Right Coronary Artery arising from the Left Anterior Descending Artery, is an extremely rare coronary artery anomaly. In a series of 126,595 coronary angiograms reported by Yanmanaka et al, it accounted for only 11 cases. $^{1}$ More recently, Wilson et $\mathrm{al}^{2}$ in a review of literature reported 36 published cases describing this type of anomaly. In most cases, the origin of the anomalous RCA is from the proximal to mid portion of the LAD. ${ }^{3-5}$ Usually benign, anomalous RCA from LAD may occasionally cause ischemia by various mechanisms. These include atherosclerosis frequently present at the proximal points of branching, ${ }^{6}$ or reduced flow velocities in the anomalous vessel due to acute angle of take off. ${ }^{7}$

Few case reports of percutaneous intervention for revascularisation in this type of coronary anomaly exist. Of the 36 cases reviewed by Wison et al, ${ }^{2} 4$ underwent PTCA and stenting. ${ }^{8-11}$ In each of these, the LAD was stented. Apart from our case, only two cases of bifurcation stenting to anomalous RCA/LAD have been reported till date. Das et $\mathrm{al}^{12}$ first reported percutaneous intervention in bifurcation lesion of anomalous RCA/LAD. They performed rotational atherectomy to debulk the bifurcation followed by simultaneous kissing stenting to RCA and LAD. Khan et $\mathrm{al}^{13}$ recently reported another case of bifurcation stenting in anomalous RCA/LAD with the Culotte technique. Our case differed from these two cases in respect of the bifurcation stenting technique used. In our patient, the difficulties associated with revascularisation included a large myocardium at risk as well as the sharp angle between the RCA and LAD. The lesion was a Medina 1,1,1 lesion with the side branch in fact being a dominant RCA. An elective two stent approach was adopted using the mini crush technique. As the acute angle between the LAD and anomalous RCA posed difficulty in passing the balloon in the RCA, a GuideLiner catheter was used to facilitate its passage and subsequent 
angioplasty and stenting.

\section{CONCLUSION}

An anomalous RCA from LAD is an uncommonly encountered coronary artery anomaly. It poses significant challenges to percutaneous intervention due to tortuous course, sharp angulations and a large area of myocardium at risk. However, with the use of unconventional hardware for optimum access and support, bifurcation stenting of an anomalous coronary artery can be performed successfully. We found the use of GuideLiner catheters in particular to be of great help in providing support and facilitating the passage of balloon and stent to the anomalous Right Coronary artery.

\section{CONFLICT OF INTEREST:}

None to declare.

\section{FUNDING INFORMATION:}

There was no external funding in the preparation of this manuscript.

\section{ETHICAL APPROVAL:}

Informed consent was obtained from the patient.

\section{ACKNOWLEDGEMENTS:}

None.

\section{AUTHOR CONTRIBUTION:}

HKB: first operator in the case, planned and performed the procedure and took decisions on hardware and technique used. AD: assisted in procedure and drafted the manuscript. SB: assisted in procedure. All authors read, revised and approved the final manuscript.

\section{REFERENCES}

1. Yanmanaka O, Hobbs RE. Coronary artery anomalies in 126,595 patients undergoing coronary angiography. Cathet Cardiovasc Diagn 1990;21:28-40

2. Wilson J, Reda H, Gurley JC. Anomolous right coronary artery originating from the left anterior descending artery: case report and review of the literature. Int J Cardiol 2009;137:195-198.

3. Erdogan O, Buyuklu M, Aktoz M. Anomalous origin of the right coronary artery from the left anterior descending artery in a patient with single left coronary artery: a rare coronary artery anomaly and review of the literature. Int J Cardiol 2008;127:280-283.

4. Meric M, Demircan S, Pinar M, Yuksel S. Single coronary artery: right coronary artery originated from middle of left anterior descending artery in a patient with severe mitral regurgitation. Clin Cardiol 2010;33:E48 E50.

5. Balghith M. Anomalous origin of the right coronary artery from the proximal left anterior descending artery and a single coronary artery anomaly: three case reports. J Saudi Heart Assoc 2013;25:43-46

6. Halon DA, Sapoznikov D, Lewis BS, Gotsman MS. Localization of lesions in the coronary circulation. Am J Cardiol 1983;52:921-6.

7. Amasyali B, Kursaklioglu H, Kose S, Iyisoy A, Kilic A, Isik E. Single coronary artery with anomalous origin of the right coronary artery from the left anterior descending artery with a unique proximal course. Jpn Heart J 2004;45:521-5.

8. Calabrò P, Bianchi R, Palmieri R, Sordelli C, Bigazzi MC, Calabrò R. Evidence of right coronary from mid-left anterior descending coronary: a rare case of coronary anomalous origin. Eur Heart J 2008;10.

9. Ocal A, Kilci H, Altunkas F, Tumuklu MM. Successful percutaneous coronary angioplasty in a patient with anomalous origin of the right coronary artery from the left anterior descending artery. Int $\mathrm{J}$ Cardiol 2008;127:e42-4. 
10. Takano M, Seimiya K, Yokoyama S, et al. Unique single coronary artery with acute myocardial infarction: observation of the culprit lesion by intravascular ultrasound and coronary angioscopy. Jpn Heart J 2003;44:271-6.

11. Dewilde W, Ten Berg JM, Scholzel B. An anomalous right coronary artery originating from the mid portion of the left descending artery. Int J Cardiol 2008;134:e68-9.

12. Das PK, Meredith IT. Rotational Atherectomy and Simultaneous Kissing Stenting of a Bifurcation Stenosis of Left Anterior Descending and Anomalous Right Coronary Arteries. Heart, Lung and Circulation. 2006 Oct 1;15(5):342-5.

13. Khan UA, Sharma D, McGlinchey P, Peace A. Percutaneous coronary intervention to left anterior descending artery/right coronary artery bifurcation: this is not a typo! A case report. European Heart Journal-Case Reports. 2019 Sep;3(3):ytz137.

\section{FIGURE LEGENDS:}

- Figure 1: Left coronary angiogram showing a heavily calcific LAD with ostio-proximal patent LAD stent and diffuse 70-80\% stenosis in mid LAD, a dominant RCA with anomalous origin from mid LAD and ostial 90\% stenosis (Medina 1,1,1 disease at the mid LAD and anomalous RCA bifurcation).

- Figure 2: The RCA lesion crossed with a 0.14 inch BMW universal wire (Abbott Vascular, Santa Clara, USA) with its distal end parked in the PDA. 2.5x15mm Sprinter balloon (Medtronic Inc, USA) advanced into anomalous RCA using a 6F GuideLiner catheter (Vascular Solutions, MN, USA) and predilatation done

- Figure 3: A Xience Xpedition 2.75x15mm stent (Abbott Vascular, Santa Clara, USA) positioned in proximal RCA and with slight protrusion into the LAD and deployed at 12 atmospheric pressure

- Figure 4: RCA stent crushed with a 2.75x18mm Sprinter balloon (Medtronic Inc, USA)

- Figure 5: 3x23mm Xience Xpedition stent (Abbott Vascular, Santa Clara, USA) placed in mid LAD and deployed at 12 atmospheric pressure

- Figure 6: After recrossing the RCA stent with a Fielder XT wire (Asahi Intecc, Nagoya, Japan, 6F GuideLiner catheter (Vascular Solutions, MN, USA) used to facilitate passage of balloon and serial dilatation of ostium of RCA done

- Figure 7: Final kissing balloon post dilatation done with two non compliant balloons, Voyager NC 3x15mm (Abbott Vascular, Santa Clara, USA) in mid LAD and Voyager NC 2.75x15mm (Abbott Vascular, Santa Clara, USA) in RCA at 20 atmospheric pressure

- Figure 8: After POT, a satisfactory angiography result with TIMI 3 flow in both LAD and RCA seen with no residual stenosis and no dissection 


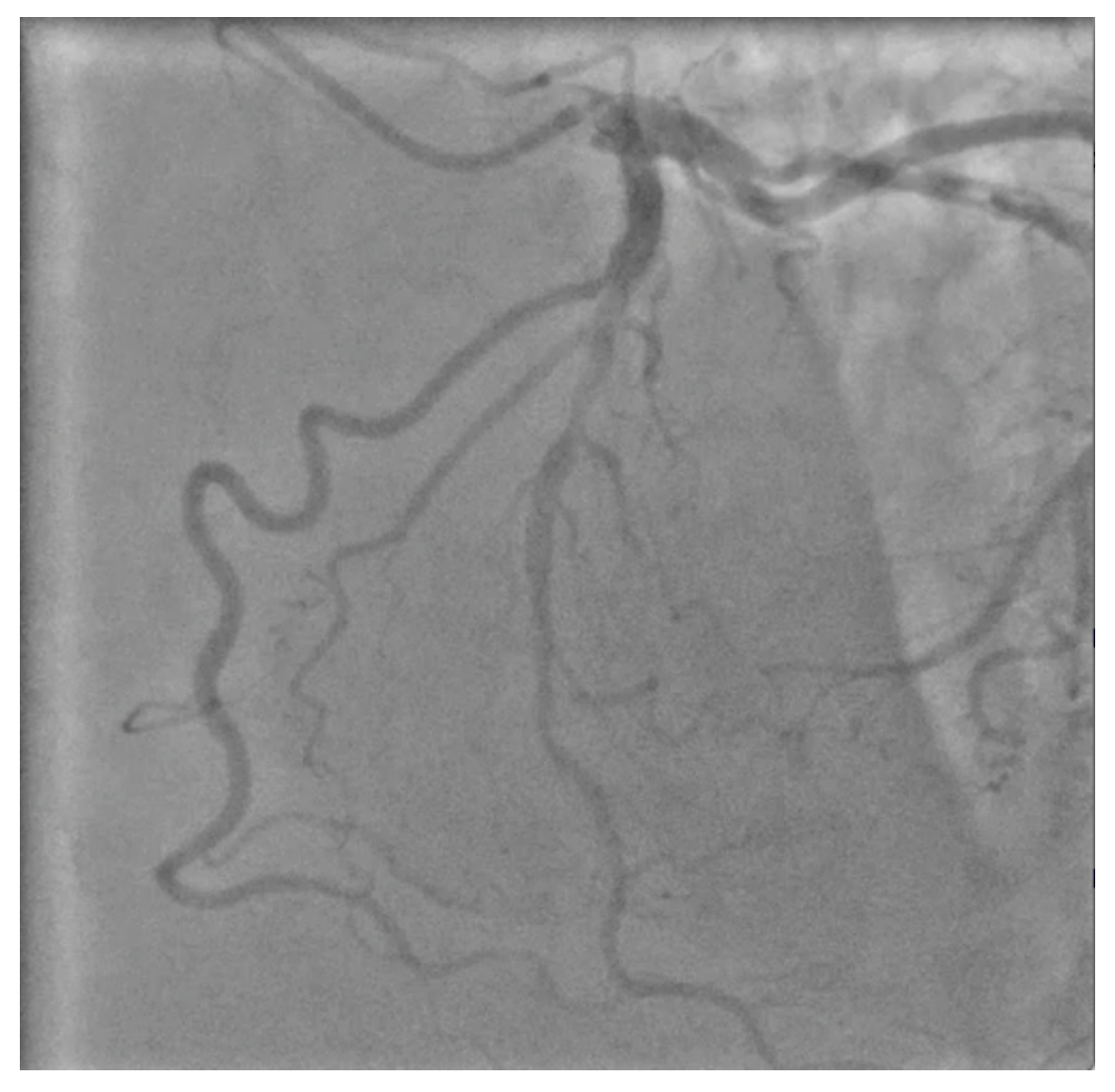




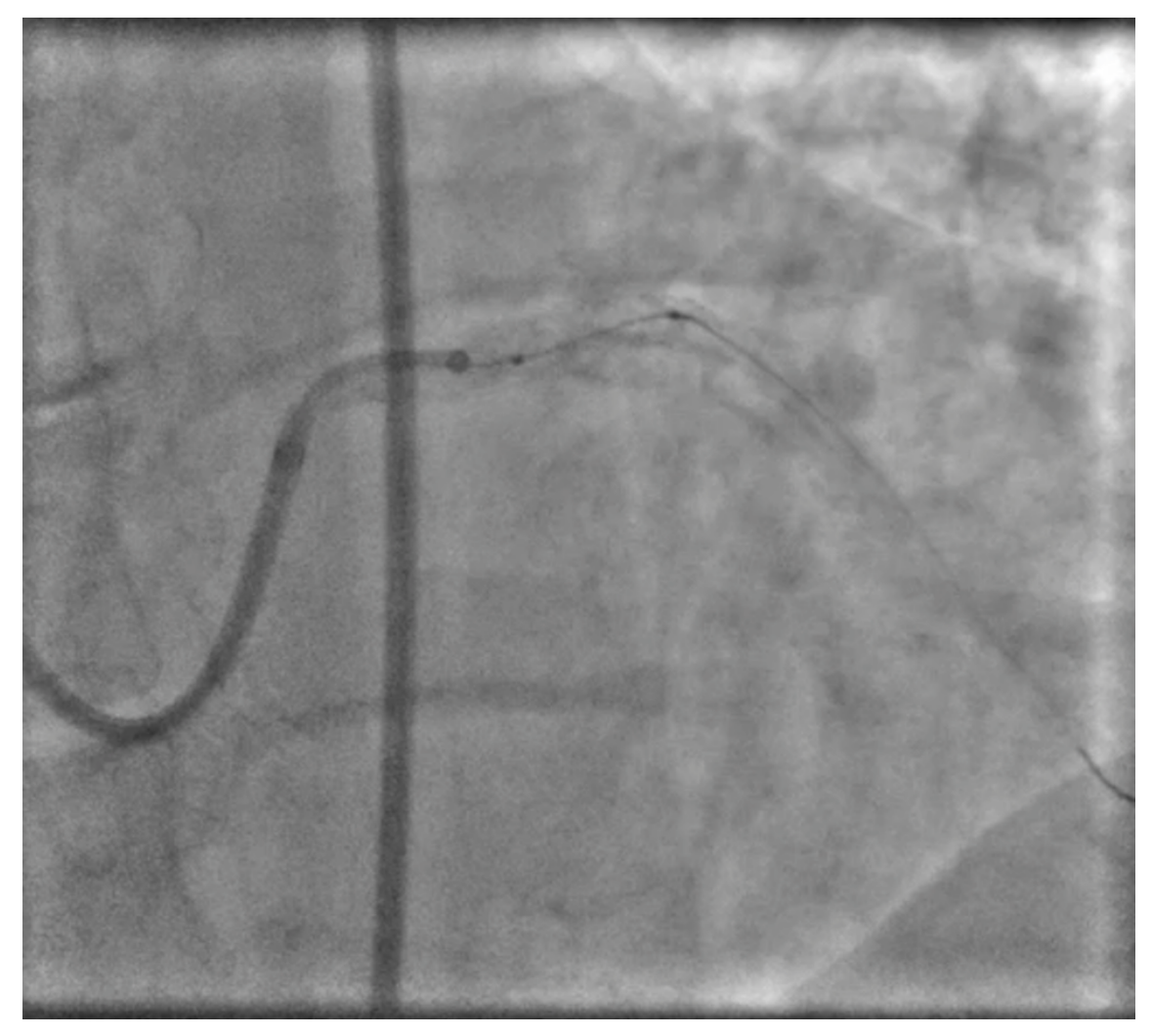




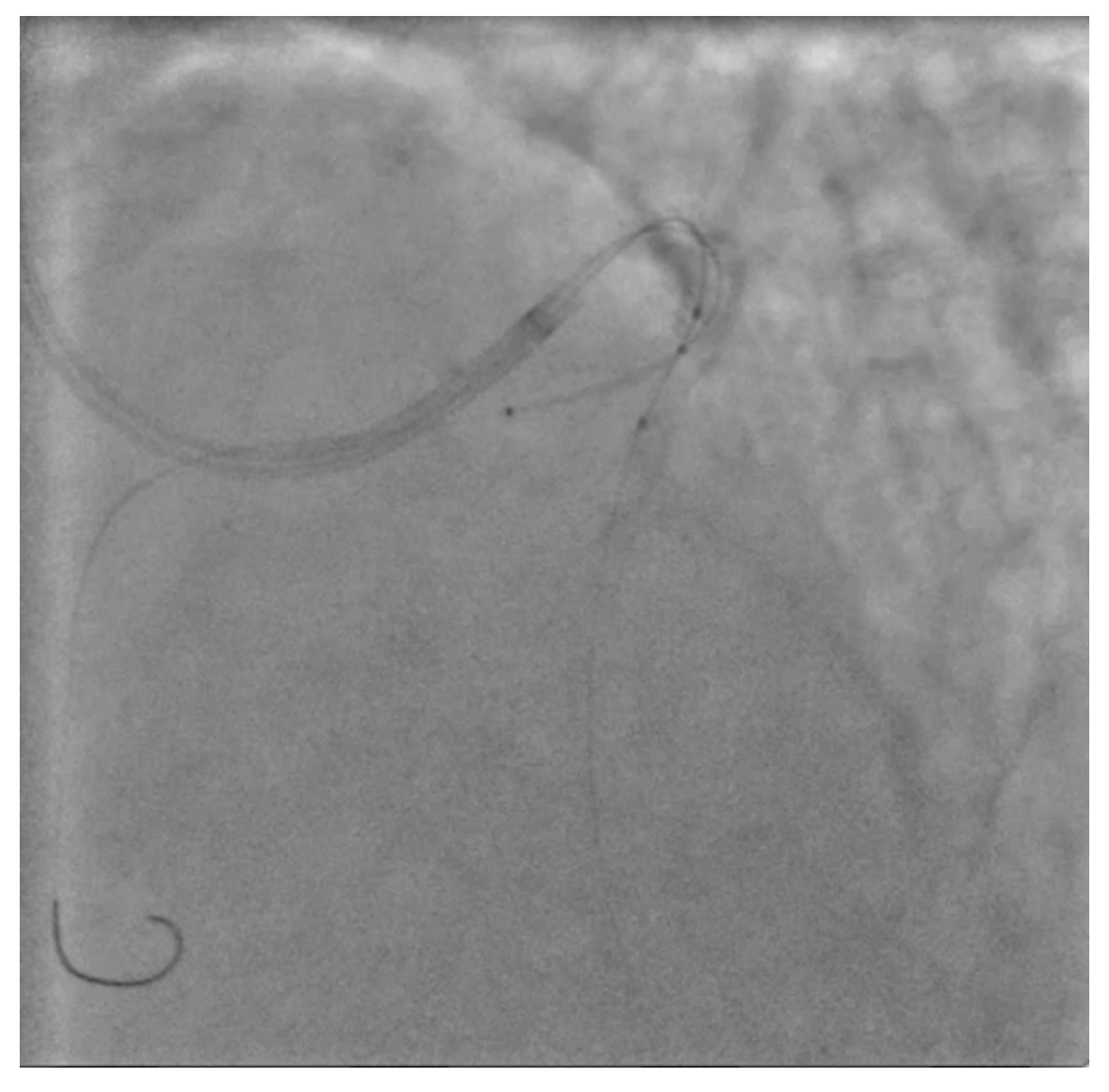




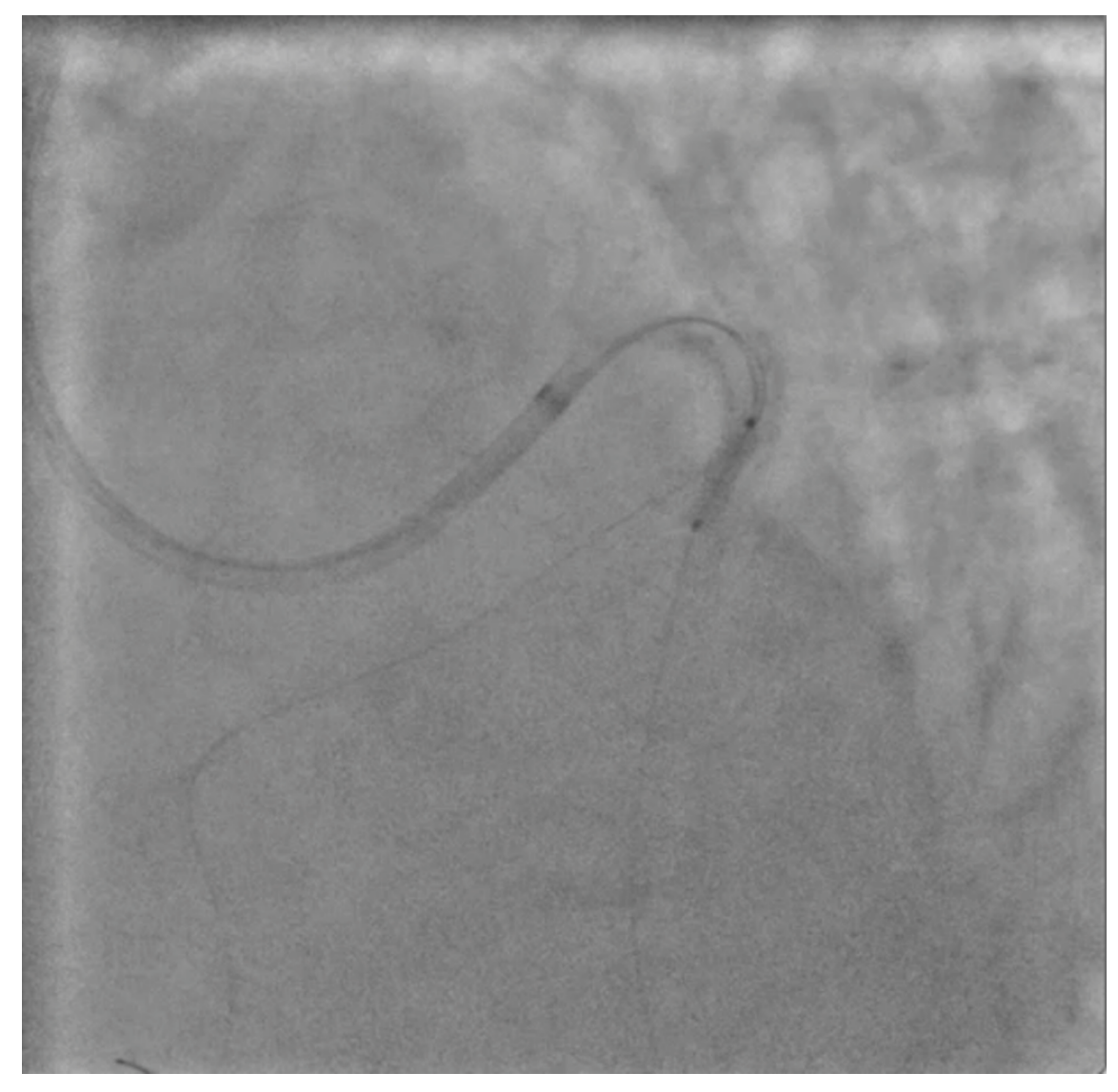




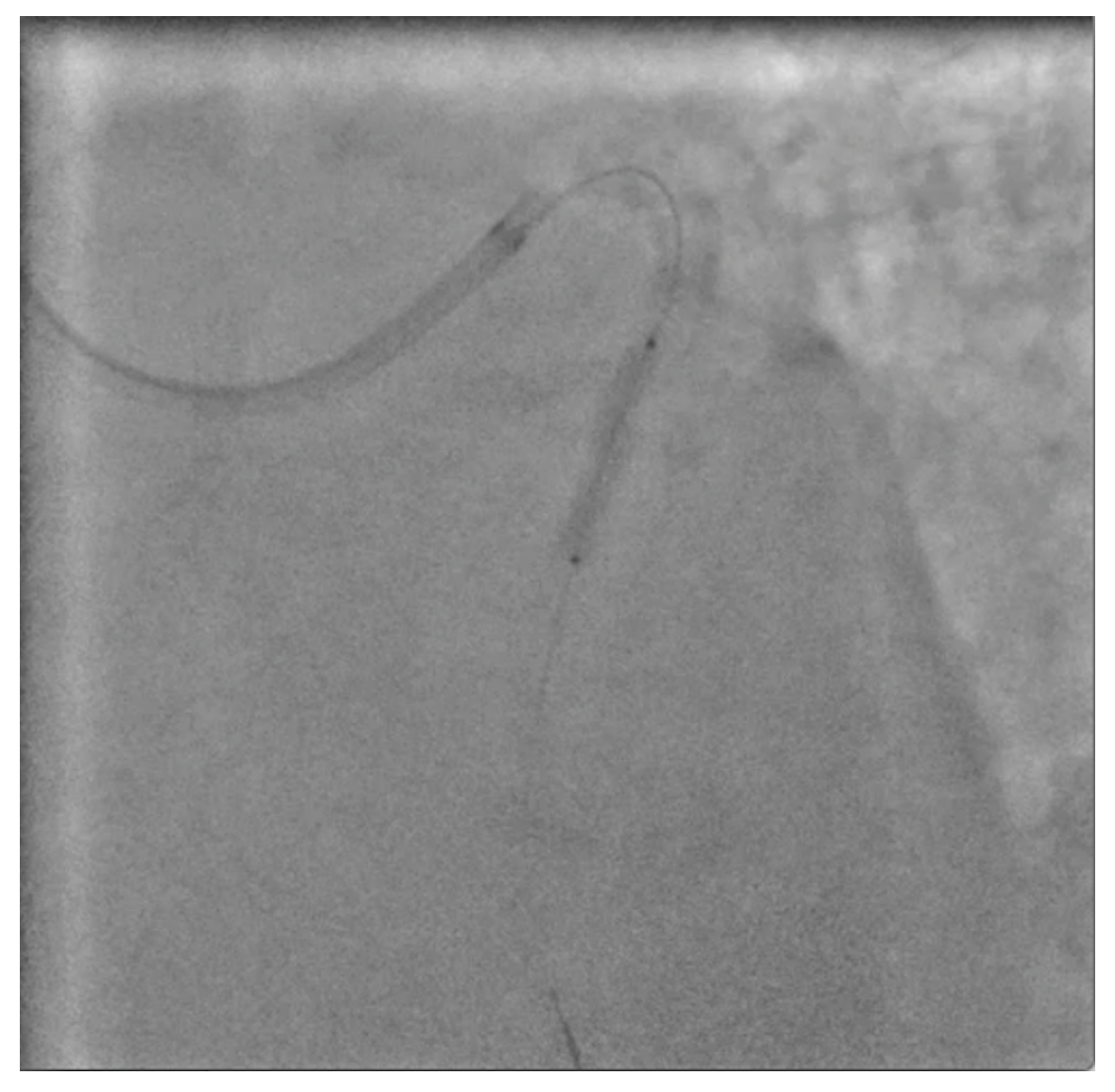




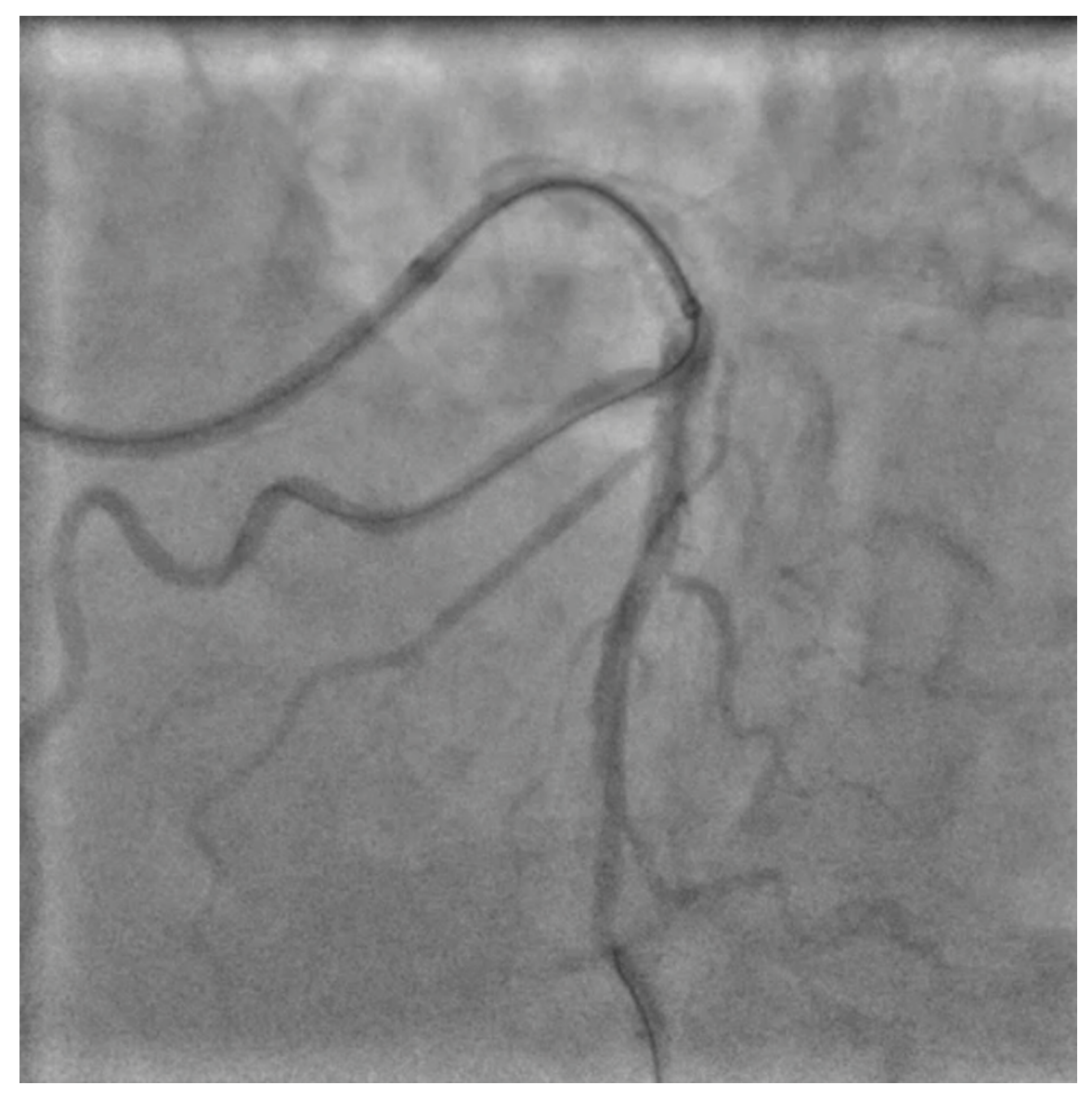




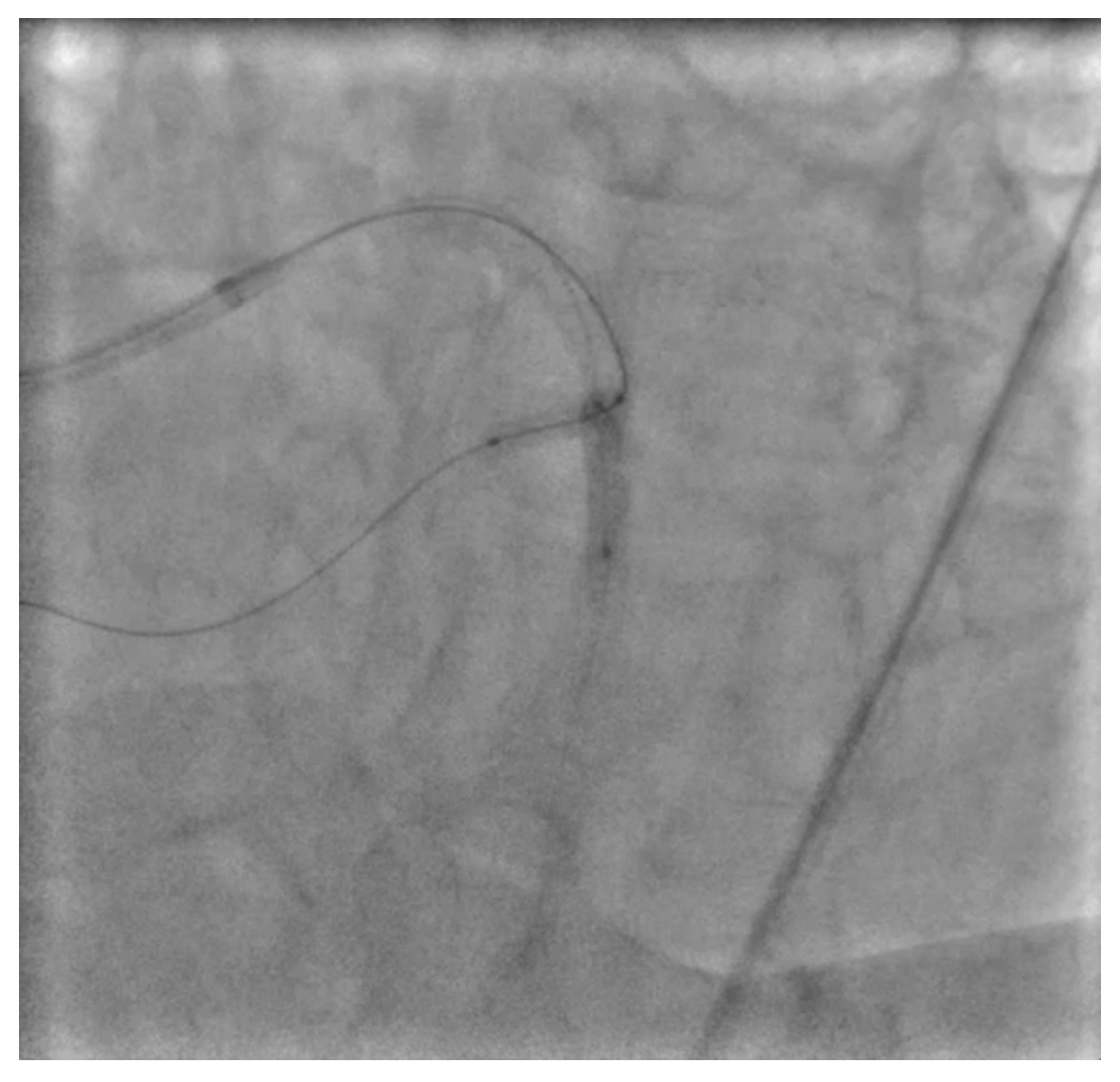




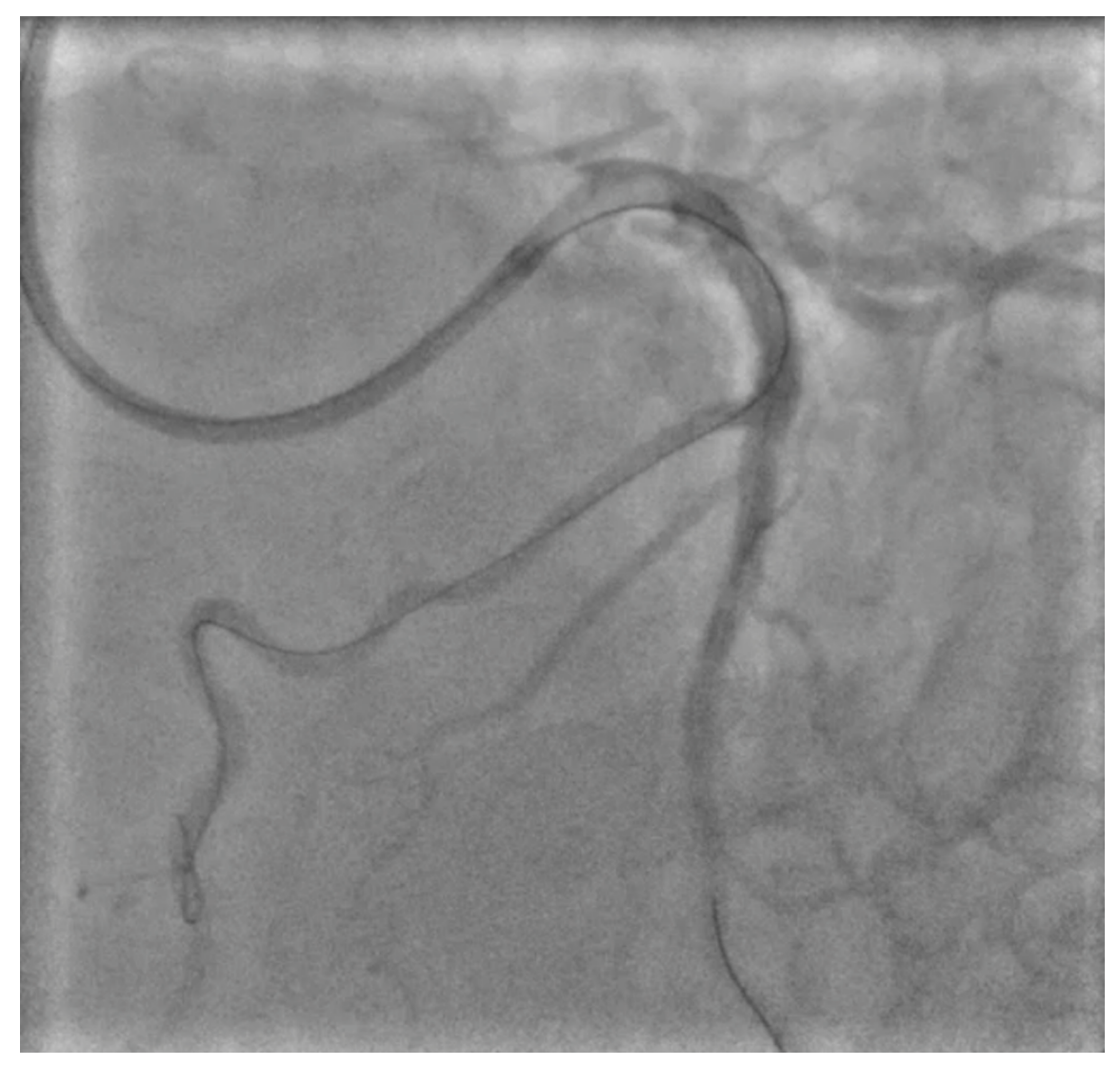

